\title{
TRANSCUTANEOUS PARTIAL OXYGEN TENSION AND SKIN BLOOD FLOW MONITORING: CONTINUOUS, NONINVASIVE MEASURES OF CARDIORESPIRATORY CHANGE *
}

\author{
Mark W. GREENLEE and Munehira AKITA ** \\ Neurologische Klinik mit Abteilung fur Neurophysiologie, 7800 Freiburg, F.R.G.
}

Accepted for publication 17 September 1984

Transcutaneous partial oxygen tension (tc $\mathrm{pO}_{2}$ ) and skin blood flow (via heat clearance) were measured noninvasively in 22 male subjects who performed stress-inducing tasks (i.e. hand-grip exercise, cold pressor test, breath holding, hyperventilation and mirror-tracing). An analysis of variance and covariance was conducted for $\mathrm{tc} \mathrm{pO}_{2}$, heat clearance, heart rate, respiration rate, finger pulse volume and systolic/diastolic blood pressure. Results indicate that tc $\mathrm{pO}_{2}$ can depict phasic cardiorespiratory challenge. Heat clearance proved to be less sensitive to the demand conditions used here. An analysis of covariance revealed a negative correlation between tc $\mathrm{pO}_{2}$ and blood pressure and respiration rate, as well as a positive correlation between tc $\mathrm{pO}_{2}$ and heat clearance. This suggests that phasic changes in tc $\mathrm{pO}_{2}$ are induced by both peripheral vasomotor activity and changes in arterial $\mathrm{pO}_{2}$.

\section{Introduction}

Cardiovascular psychophysiology is concerned with the physiological responses and response patterns elicited in human subjects during stress-inducing challenge (Ax, 1953; Fahrenberg, Walschburger, Foerster, Myrtek and Müller, 1983; Lacey, 1967). Since most experimental paradigms require healthy subjects to perform various tasks, physiological parameters should be measured noninvasively, because invasive measures could impair the subject's ability to respond and may entail certain risks. This limits researchers to a modest number of physiological measurements.

Though an important indicator of cardiorespiratory functioning, blood gas analysis has not been systematically conducted by psychophysiologists, be-

* A preliminary report on part of this research was presented at the Experimental Psychology Society Meeting, June 30-July 2, 1982 in St Andrews, Scotland and at the Meeting of the German Physiological Society, March 28-30, 1984 in Dortmund, West Germany.

** On leave of absence from: Kyoto Institute of Technology, Psychological Laboratories, 606 Kyoto, Japan. 
cause it requires invasive methods. In addition, the procedure of blood taking is a challenge in and of itself. Such a measurement, therefore, may confound any effect due to experimental manipulation.

\section{Polarographic measurement of transcutaneous $\mathrm{pO}_{2}$}

A method to estimate partial arterial oxygen pressure $\left(\mathrm{a} \mathrm{O}_{2}\right)$ through the skin, termed transcutaneous partial oxygen tension $\left(\mathrm{tc} \mathrm{pO}_{2}\right)$, has been developed by Lübbers and his co-workers (Huch, Lübbers and Huch, 1972, Lübbers, 1981). Based on the polarographic measurement of oxygen in a liquid via the Clark method (Clark, 1956), a $\mathrm{pO}_{2}$ can be estimated at the skin surface. To do this, the skin surface must first be heated to $44^{\circ} \mathrm{C}$ so that a state of blood flow independency is reached. The resulting hyperaemia provides the dermis with a rich amount of arterialized blood, thereby saturating the oxygen needs of the skin. The polarographic sensor has three platinum cathodes that are submerged in an electrolyte solution. Oxygen passes through a telfon-cellophane membrane covering the transducer and changes the potential of these cathodes.

To monitor skin blood flow, the amount of heat clearance can be directly measured by recording the electrical current needed to maintain a fixed temperature at the electrode. Since the temperature of the electrode is greater than that of blood, blood flow dissipates the heat of the electrode. It follows that the greater the blood flow the more current will be needed to maintain the electrode's temperature. Thus, a second measure is produced by the same electrode, namely, skin blood flow via heat clearance.

Cardiovascular physiologists and anesthesiologists have been concerned with tc $\mathrm{pO}_{2}$ as a correlate of a $\mathrm{pO}_{2}$. Lübbers (1981) has demonstrated that under controlled conditions, tc $\mathrm{pO}_{2}$ can reflect arterial $\mathrm{pO}_{2}$ if blood flow independency is achieved. Huch, Huch and Lübbers (1982) monitored tc $\mathrm{pO}_{2}$ and intra-arterial a $\mathrm{pO}$, in subjects given $95 \% \mathrm{O}$, and $5 \% \mathrm{CO}$. Their subjects further performed hyperventilation and breath holding. The correspondence between the resulting tc $\mathrm{pO}_{2}$ and a $\mathrm{pO}_{2}$ records is quite impressive, but correlation coefficients were not reported.

The present study has been conducted to explore the value of transcutaneous partial oxygen tension (tc $\mathrm{pO}_{2}$ ) and skin blood flow monitoring in subjects exposed to typical laboratory challenge. These noninvasive microcirculatory measures should prove to be interesting variables for psychophysiologists because changes in tc $\mathrm{pO}_{2}$ reflect changes both in arterial $\mathrm{pO}_{2}$ and in skin blood flow due to vasoconstriction. As such, tc $\mathrm{pO}_{2}$ and heat clearance give an index of cardiorespiratory functioning.

If tc $\mathrm{pO}_{2}$ and skin blood flow measures are to be useful, they must first be tested to see whether they are sensitive enough to reflect changes induced by demanding tasks. Therefore, we conducted measurements over a number of experimental conditions to first assess the sensitivity of tc $\mathrm{pO}_{2}$ and heat 
clearance to various types of cardiorespiratory challenge. Significant differences from resting levels should be evident during adequate challenge. We also compared tc $\mathrm{pO}_{2}$ and heat clearance values with those of traditional variables used in cardiovascular psychophysiology.

\section{Method}

\subsection{Subjects}

Male student volunteers ( $N=22$, mean age, 26 yrs, $\mathrm{SD}=2.3)$ free of any obvious illness participated in the experiment which lasted about two hours. They either received credit fulfilling requirements for their studies in psychology or a fee ( $\$ 8.00$ US). Subjects were asked to abstain from caffeine or alcoholic beverages and tobacco for a three-hour period prior to examination. All subjects were right-handed.

\subsection{Apparatus}

We used the Oxymonitor (Hellige $\mathrm{GmbH}$, Freiburg) to measure tc $\mathrm{pO}_{2}$ and heat clearance. The oxygen sensor was calibrated to room air with reference to local barometric pressure. Following ten minutes of stable values, the sensor was positioned on the skin surface of the inside of the left forearm. A contact agent (Hellige, $\mathrm{GmbH}$ ) and proper adhesive rings assured a direct interface between the skin and electrode. The electrode then hyperaemized the cutaneous tissue within a $15 \mathrm{~min}$ period.

The following parameters were also measured continuously during all rest and challenge phases: Heart rate, respiration rate ( 3 ECG chest leads), finger pulse volume (amplitude recorded on the left index finger using pneumatic plethysmography without venous occlusion, Boucker pressure transducer). Data were recorded on an 8-channel polygraph and analyzed off-line with a sampling rate of $20 / \mathrm{min}$. Data were reduced to mean values for each subject on each measure in the given condition: These mean values were then used in the analyses of variance and covariance.

Additionally, blood pressure was measured using a manometer on the right arm directly following each challenge phase (except between hyperventilation and breath holding). A cuff was manually inflated to $170 \mathrm{mmHg}$ and a microphone positioned over the antecubital artery picked up the Korokoff sounds.

\subsection{Procedures}

\subsubsection{Electrode placement and calibration}

Subjects were asked to enter the air-conditioned chamber $\left(25 \pm 1^{\circ} \mathrm{C}\right.$, relative humidity 55\%) and were seated in a comfortable chair. Instructions were given 
concerning the nature of the tasks and the physiological measures to be made. After subjects gave their consent, electrodes were positioned and calibrated. Adequate time $(15 \mathrm{~min}$ ) was then given to allow for hyperaemization of the skin area beneath the electrode for the tc $\mathrm{pO}_{2}$ recording. Subsequently, a five-minute baseline recording was conducted.

\subsubsection{Cardiorespiratory challenge}

First, various physical challenges were induced to test cardiorespiratory response to standardized stimulation. These were: (1) Ergometric exercise (self-paced hand-grip exercises lasting $2 \mathrm{~min}$ ); (2) cold pressor (left forearm submerged in $4^{\circ} \mathrm{C}$ water which was stirred by a magnetically coupled propeller to prevent a warm layer from forming around the arm's surface, 1 min); (3) self-paced hyperventilation ( $1 \mathrm{~min})$; (4) breath holding (at inspiratory level for as long as possible; maximal duration of $2 \mathrm{~min}$ ). Following each challenge, subjects were allowed a 2 -min rest period similar to baseline recording. 'The sequence of conditions was kept constant to allow analysis of between-subjects variance (see Data Analysis). Sufficient time for recovery minimized any carry-over effects.

\subsubsection{Sensorimotor tasks}

Subsequent to these challenges, subjects were instructed as to the nature of the sensori-motor test. It consisted of four mirror-tracing tasks of increasing difficulty. We used the Sensorimotor Training instrument (Zak Company, Munich) together with a tilt mirror and occluder. During tracing, whenever subjects traced outside of the pattern an error was signalled. The error was recorded and simultaneously a tone was produced to inform the subject of the error. To increase the competitive nature of the tracing tasks, performance norms, based on preliminary testing, were presented. Subsequent to performance, subjects were informed about their scores, which they then could compare to these norms. Tracing tasks were employed because they require concentration and do not require verbal responses, which would interrupt breathing and, thus, induce artifactual changes in tc $\mathrm{pO}_{2}$.

\subsection{Data analysis}

\subsubsection{Analysis of variance}

An analysis of variance was used to test the main effects 'conditions' and 'subjects'. If tc $\mathrm{pO}_{2}$ and heat clearance are sensitive measures, then challenges eliciting changes in cardiorespiratory functioning should produce significant changes in these variables, thus yielding a significant main effect for 'conditions'. If, however, tc $\mathrm{pO}_{2}$ and heat clearance values exhibit considerable individual differences, then the main effect 'subjects' should be significant. 
The amount of variance accounted for by these terms was used to indicate the degree to which these variables were comparable to traditional measures. In addition, the error term was assessed as a measure of reliability (a low error term corresponds to good reliability). The Scheffé test (Scheffé, 1953) is employed as a conservative test of significant pair-wise differences between initial rest and strain values.

\subsubsection{Analysis of covariance}

An analysis of covariance (see, Fahrenberg and Foerster, 1982) was conducted to assess the degree to which tc $\mathrm{pO}_{2}$ and heat clearance covary with standard measures of cardiorespiratory function during rest and challenge. The between-subjects coefficients were based on the reduced values on each variable averaged over all rest and challenge conditions for each subject separately. The resulting coefficients provided an index of covariance between variables after the differential effect of the various conditions had been partialled out by averaging each subject's value over the ten conditions. These coefficients were submitted to normal tests of significance with $\mathrm{df}=21$.

\section{Results}

Fig. 1 presents the mean values and standard errors across subjects for each physiological measure during the ten experimental conditions. As can be seen in fig. 1, tc $\mathrm{pO}_{2}$ increases during hand-grip exercise, hyperventilation and tracing tasks, whereas breath holding induces decreases. Heat clearance, on the other hand, fails to demonstrate significant changes from initial baseline values.

Table 1 presents the results of the analysis of variance for the main effects 'conditions' and 'subjects'. The columns (from left to right) present the sources of variance, the sums of squares, the degrees of freedom, the mean square, the $F$ ratio, the significance of the $F$ ratio, and the amount of variance accounted for by the term in question. In addition, the results of the Scheffé test for significant differences from initial resting values are given in the final column.

As can be seen in table 1, the $F$ ratios for the main effects 'conditions' and 'subjects' are highly significant for most variables. The exceptions are the main effect 'conditions' for heat clearance $(p=0.038)$ and for diastolic blood pressure ( $p=0.137 ; \mathrm{ns})$. Of more interest is the amount of variance accounted for by either of the main effects. For tc $\mathrm{pO}_{2}$, the main effect 'subjects' accounts for $60 \%$ of the total variance, whereas the main effect 'conditions' accounts for only $12.8 \%$. For heat clearance, 'subjects' accounts for $84.1 \%$ and 'conditions' merely for $1.8 \%$ of the total variance. Compared to heart rate $(46.1 \%$ and $28.2 \%$ for 'subjects' and 'conditions', respectively), tc $\mathrm{pO}_{2}$ and heat clearance are less sensitive to phasic changes induced by the challenges used here. 


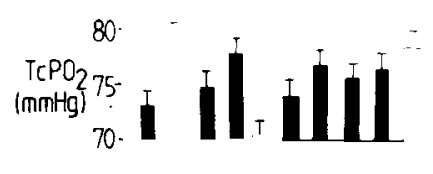

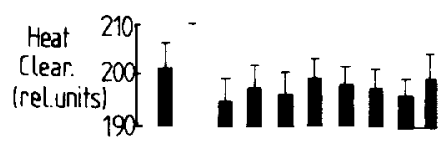
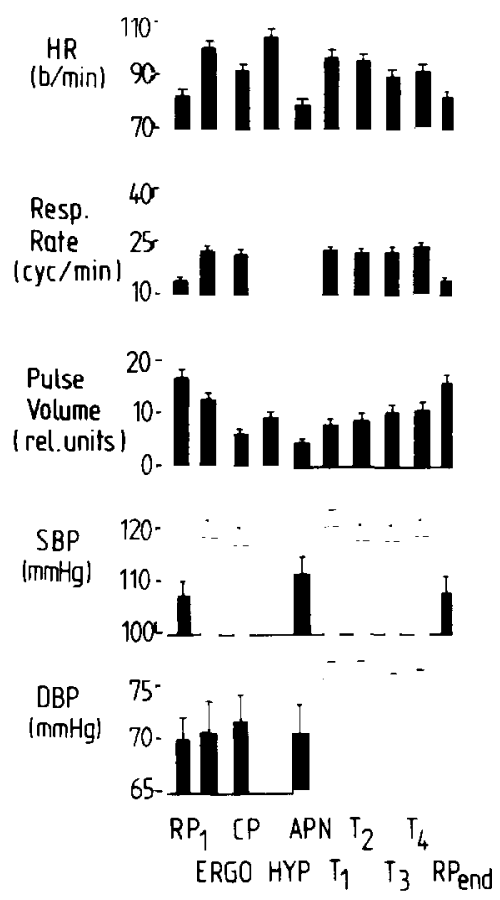

Fig. 1. Mean values and standard errors across subjects on physiological measures recorded during each experimental condition. Abbreviations: RP1 = rest period 1; ERGO = ergometric exercise; $\mathrm{CP}=$ cold pressor test; HYP = hyperventilation; $\mathrm{APN}=$ breath holding; $\mathrm{T} 1-\mathrm{T} 4=$ tracing tasks 1-4; $\mathrm{RPend}=$ final rest period; $\mathrm{HR}=$ heart rate; $\mathrm{SBP}=$ systolic blood pressure; $\mathrm{DBP}=$ diastolic blood pressure.

The results of the Scheffé tests further support these findings. Whereas tc $\mathrm{pO}_{2}$ values recorded during ergometric exercise and during the final rest phase significantly differ from initial rest values, heat clearance values recorded during the various challenges and final resting do not significantly differ from initial values.

Table 2 depicts between-subjects correlation coefficients for each physiological measure. These coefficients are based on each subject's averaged values 


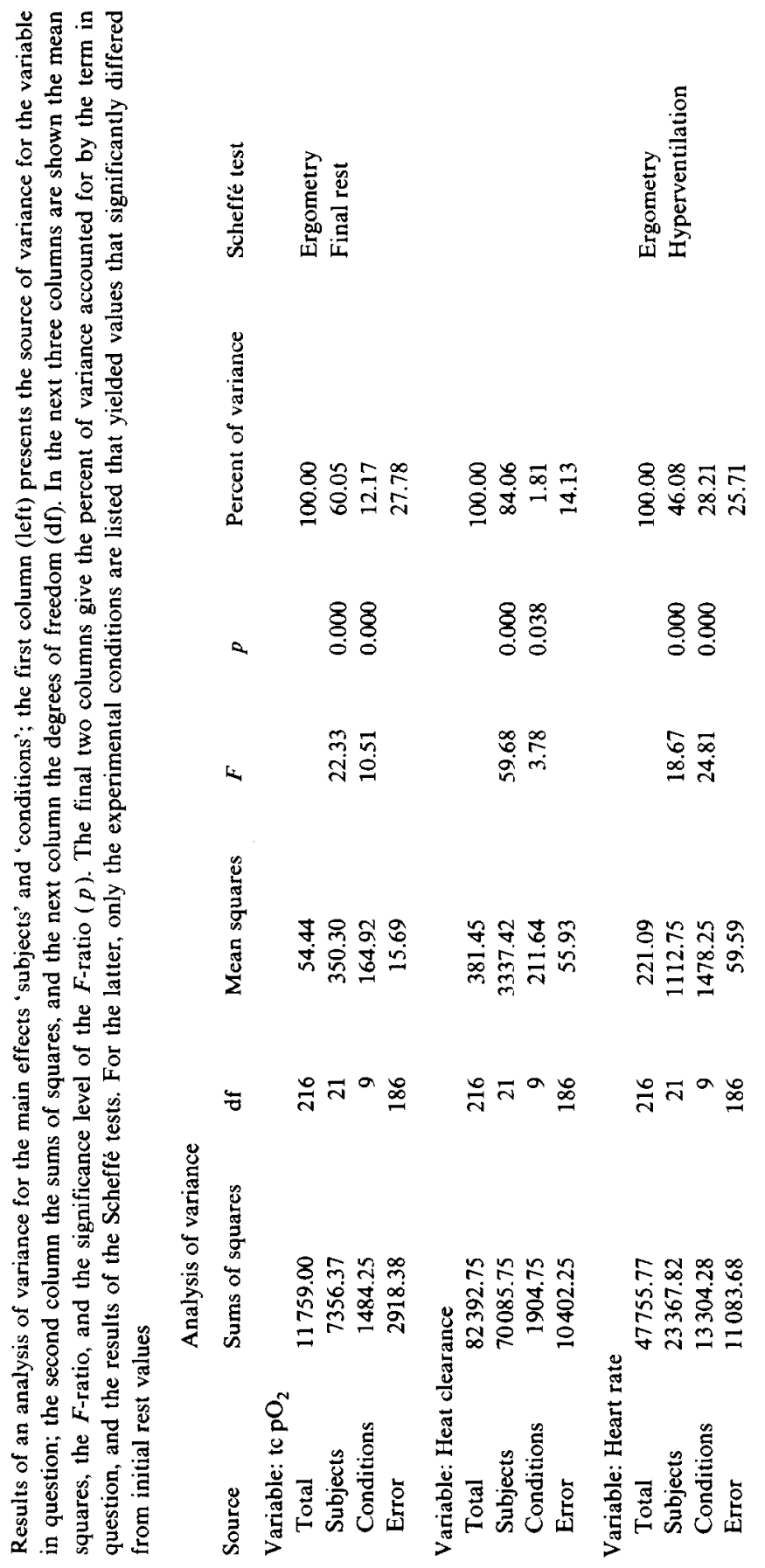



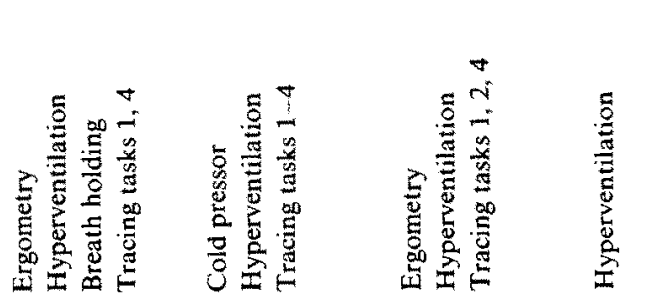

$\frac{2}{3}$

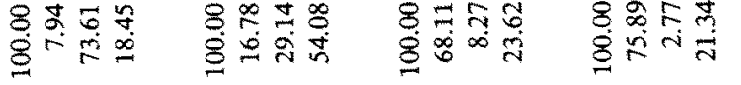

홍형

s:

옹영

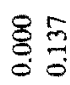

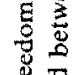

$\pm \frac{n}{n}$

88
80
0

in

$\because 8$

号

总

용

莡

政

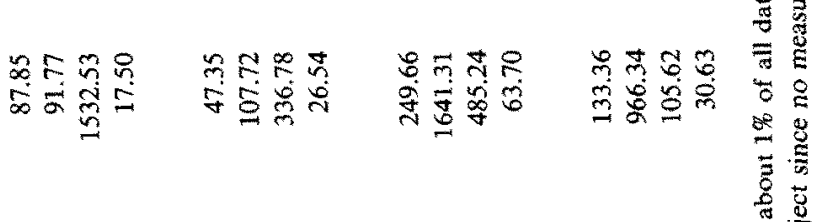

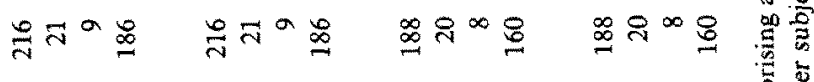

됴유.

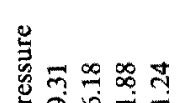

莺

逭

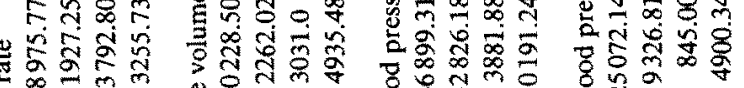

${ }_{\infty}^{\infty}=$

范

䨪

弯营

总

$\overline{8} \underset{\infty}{\infty} \stackrel{\infty}{\infty}$

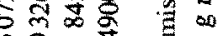

要恋

8

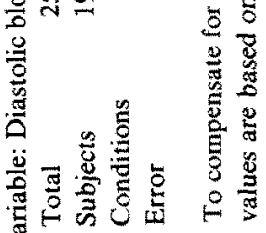


Table 2

Between-subjects covariance coefficients are presented for the physiological measures recorded. These are based on values averaged for each subject over the ten experimental conditions; $\mathrm{df}=21$. Significance levels: $r=0.41, p=0.05 ; r=0.54, p=0.01$

$\begin{array}{llllllll}1 & 2 & 3 & 4 & 5 & 6 & 7\end{array}$

(1) $\mathrm{Tc} \mathrm{pO}_{2}$

(2) Heat clearance $\quad 0.37$

(3) Heart rate $\quad-0.35$

(4) Respiration $\quad-0.39$

(5) Pulse volume $\quad 0.00$

(6) Systolic BP $\quad-0.40$

(7) Diastolic BP $\quad-0.42$

$$
\begin{array}{rrr}
-0.09 & & \\
-0.13 & 0.25 & \\
-0.02 & -0.05 & -0.31 \\
-0.30 & 0.46 & 0.13 \\
-0.29 & 0.56 & 0.04
\end{array}
$$$$
\begin{array}{lll}
-0.30 & 0.46 & 0.13
\end{array}
$$

0.04

$0.17 \quad 0.74$

from the 10 experimental conditions. These coefficients reflect the degree to which between-subject differences covary after eliminating the various effects of the demanding tasks by averaging.

As can be seen in table 2, covariance between physiological measures is generally modest to low. The main exceptions are found between tc $\mathrm{pO}_{2}$ and heat clearance $(0.37)$, tc $\mathrm{pO}_{2}$ and respiration rate $(-0.39)$, tc $\mathrm{pO}_{2}$ and systolic blood pressure $(-0.40)$, tc $\mathrm{pO}_{2}$ and diastolic blood pressure $(-0.42)$ and systolic/diastolic blood pressure and heart rate ( 0.46 and 0.56 , respectively). Note that heat clearance only covaries well with tc $\mathrm{pO}_{2}$ and very little with heart rate $(-0.09)$.

\section{Discussion}

The results of the analysis of variance indicates that tc $\mathrm{pO}_{2}$ is sensitive enough to reflect changes induced by cardiorespiratory challenge. Heat clearance fails, however, to satisfactorily depict changes in skin blood flow. In addition, the analysis of variance shows that the 'subjects' term accounts for most of the variance for tc $\mathrm{pO}_{2}$ and heat clearance.

It may be argued that the 'subjects' term consists of both true individual differences and between-subjects measurement error due to differences in transducer placement, etc. However, if the variance accounted for by 'subjects' is due primarily to error, a significant correlation between tc $\mathrm{pO}_{2}$ and diastolic blood pressure $(-0.42)$ would not be expected. As this coefficient is based on the subjects' average value over all conditions, the 'conditions' variance is partialled out, thus leaving only the 'subjects' and error terms. Error variance does not covary between independent measures, which indicates that the 'subjects' term does consist of meaningful variance.

To further substantiate between-subjects differences, a replication with the same subject sample using identical conditions would, in some respects, be 
preferable, but not completely unproblematic. Experiencing the same challenges for a second time, subjects would surely respond differently. Therefore, responses would vary depending on how well subjects habituate to the conditions.

In summary, the present findings suggest that tc $\mathrm{pO}_{2}$ can depict changes elicited by cardiorespiratory challenge. Heat clearance is not, however, sensitive enough to depict changes in skin blood flow induced by the challenges administered here. The significant amount of variance accounted for by the 'subjects' term and the significant correlation with diastolic blood pressure indicate that tonic tc $\mathrm{pO}_{2}$ values can reflect individual differences in cardiorespiratory functioning.

\section{Acknowledgements}

This research was supported by the Deutsche Forschungsgemeinschaft, SFB 70, Teilprojekt A6. Munehira Akita gratefully acknowledges financial assistance from the Deutscher Akademischer Austauschdienst (DAAD).

The authors wish to express their gratitude to $\mathrm{C}$. Wollert and $\mathrm{H}$. Leist of Hellige, GmbH. for providing use of the Oxymonitor for this research. The authors also thank Lothar Spillmann, Jochen Fahrenberg, Michael Myrtek and L.T. Sharpe for providing laboratory facilities and helpful comments to an earlier version of this manuscript. Friedrich Foerster assisted in the data analysis.

\section{References}

Ax, A.F. (1953). The physiological differentiation between fear and anger in humans. Psychosomatic Medicine, 15, 433-442.

Clark, L.C. Jr. (1956). Monitor and control of blood tissue oxygen tensions. Transactions American Society of Artificial Organs, 2, 41-48.

Fahrenberg, J. and Foerster, F. (1982). Covariation and consistency of activation parameters. Biological Psychology, 15, 151-169.

Fahrenberg, J., Walschburger, P., Foerster, F., Myrtek, M. and Müller, W. (1983). An evaluation of trait, state and reaction aspects of activation processes. Psychophysiology, 20, 188-195.

Huch, R., Huch, A. and Lübbers, D.W. (1982). Transcutaneous $\mathrm{pO}_{2}$. Thieme: Stuttgart.

Huch, R., Lübbers, D.W. and Huch, A. (1972). Quantitative continuous measurement of partial oxygen pressure on the skin of adults and newborn babies. Pflügers Archive, 337, 185-198.

Lacey, J.I. (1967). Somatic response patterns and stress: Some revisions of activation theory. In: Appley, M.H. and Trumbull, R. (Eds.). Psychological Stress: Issues in Research. AppletonCentury-Crofts: New York.

Lübbers, D.W. (1981). Theoretical basis of transcutaneous blood gas measurement. Critical Care Medicine, 9, 721-733.

Scheffé, H. (1953). A method for judging all contrasts in the analysis of variance. Biometrika, 40, 87-104. 